\title{
Synthesis of lunberger stochastic observer for estimation of the grinding operation state
}

\author{
Sergey Bratan ${ }^{1}$, and Stanislav Roshchupkin ${ }^{1, *}$ \\ ${ }^{1}$ Sevastopol State University, Department of Mechanical Engineering, 299053 Sevastopol, Russia
}

\begin{abstract}
The technique of constructing Lunberger stochastic observer for the operation of circular external grinding is considered in the article, which makes it possible to obtain optimal estimates of the output parameters of the technological system. To build Lunberger stochastic observer, a program has been developed that allows solving the Riccati equation of the object and the filter. The calculation of the Kalman-Bucy filter coefficients for the steady-state mode of the process of grinding the camshaft journal is performed. This allows us to evaluate the state of the system in real time, and even in the case of measuring only one coordinate, the filter gives estimates of all coordinates, and the maximum estimation errors do not exceed $10 \%$.
\end{abstract}

\section{Introduction}

The solution of problems of optimal control of grinding operations implies the possibility of accurate measurement of all state variables of an object and the presence of complete information about the parameters of the technological system [1-2].

\section{Main text}

Analysis of the grinding operation shows that the phase coordinates which are necessary for controlling the object are measured with significant random errors or not measured at all [47]. In this case, the movement of the control object is subject to accidental influences. Therefore, when solving management tasks, the use of deterministic methods is unacceptable [3]. In such a situation, it is necessary to have the results of evaluating the state of the technological system, which have a statistical relationship with the observational data. The dynamics of the grinding operation is described by a differential equation of the form:

$$
c \ddot{S}_{y}-a \dot{S}_{y}=-c \ddot{Q}_{\Sigma}+b \dot{Q}_{\Sigma}+\dot{y}_{T}
$$

\footnotetext{
* Corresponding author: $\underline{\text { st.roshchupkin@yandex.ru }}$
} 
where $\dot{S}_{y}$ - is the feed amount; $\dot{Q}_{\Sigma}$ - intensity of material removal; $\dot{y}_{T}$ - intensity of temperature deformations in the technological system; $a, b$ and $c$ are coefficients that take into account the compliance of the technological system.

To solve the problem of synthesizing a linear estimation (filtration) algorithm that forms an unbiased estimate of the state vector of a system with minimal variance, we represent the differential equation (1) in vector-matrix form [8-11].

Taking into account that expression (1) is a second-order differential equation, we use the standard procedure to reduce its order.

We denote by $x_{1}=Q_{\Sigma}, \dot{\mathrm{x}}_{1}=x_{2}=\dot{Q}_{\Sigma}, \dot{y}_{T}=w$, when $\dot{x}_{2}=\frac{b}{c} x_{2}+\frac{w}{c}+u$, where $u=-\ddot{S}_{y}+\frac{a}{c} S_{y}$.

Taking into account that during grinding it is possible to observe and process the amount of material removal $Q_{\Sigma}=x_{1}$, the system of equations describing the state of the object is represented in the form:

$$
\left\{\begin{array}{l}
{\left[\begin{array}{l}
\dot{x}_{1} \\
\dot{x}_{2}
\end{array}\right]=\left[\begin{array}{ll}
0 & 1 \\
0 & \frac{b(t)}{c(t)}
\end{array}\right]\left[\begin{array}{l}
\mathrm{x}_{1} \\
\mathrm{x}_{2}
\end{array}\right]+\left[\begin{array}{l}
-a(t)-b(t) c(t) \\
a^{2}(t)+a(t) b(t) c(t)
\end{array}\right] u(t)+\left[\begin{array}{c}
0 \\
\frac{1}{c(t)}
\end{array}\right] w(t),} \\
y(t)=\left[\begin{array}{ll}
1 & 0
\end{array}\right]\left[\begin{array}{l}
x_{1} \\
x_{2}
\end{array}\right]+v(t)
\end{array}\right.
$$

where $\left[\begin{array}{l}x_{1} \\ x_{2}\end{array}\right]$ - state vector of the system; $u(t)$ - control vector; $u(t)=\left[\begin{array}{c}\dot{S}_{y}(t) \\ \ddot{S}_{y}(t)\end{array}\right] ; w(t)-$ random vector; $v(t)$ - vector of random interference accompanying measurements,

$$
\begin{aligned}
& A(t)=\left[\begin{array}{ll}
0 & 1 \\
0 & \frac{b(t)}{c(t)}
\end{array}\right] ; B(t)=\left[\begin{array}{l}
-a(t)-b(t) c(t) \\
a^{2}(t)+a(t) b(t) c(t)
\end{array}\right] ; \\
& C(t)=\left[\begin{array}{c}
0 \\
\frac{1}{c}(t)
\end{array}\right] ; H(t)=\left[\begin{array}{ll}
1 & 0
\end{array}\right],
\end{aligned}
$$

- matrices corresponding to them.

Graphical interpretation of the system (2) is shown in Fig. 1.

We assume that the random effects $w$ and the interference $v$ are random Gaussian processes of the white noise type with zero mathematical expectations $M[w(t)]=0, M[v(t)]=0$, then their correlation matrices are described by the expressions:

$$
\begin{gathered}
\operatorname{cov}[w(t), \mathrm{w}(\tau)]=M\left[w(t) \mathrm{w}^{\mathrm{T}}(\tau)\right]=Q(t) \delta(t-\tau), \\
\operatorname{cov}[v(t), v(\tau)]=M\left[v(t) v^{\mathrm{T}}(\tau)\right]=R(t) \delta(t-\tau),
\end{gathered}
$$

where $\delta(t)$ - is the Dirac delta function; $Q(t)$ - is a symmetric, nonnegative definite white noise intensity matrix of the system $w(t) ; R(t)$ - is a symmetric, positively determined white noise intensity matrix of measurements $v(t)$. 


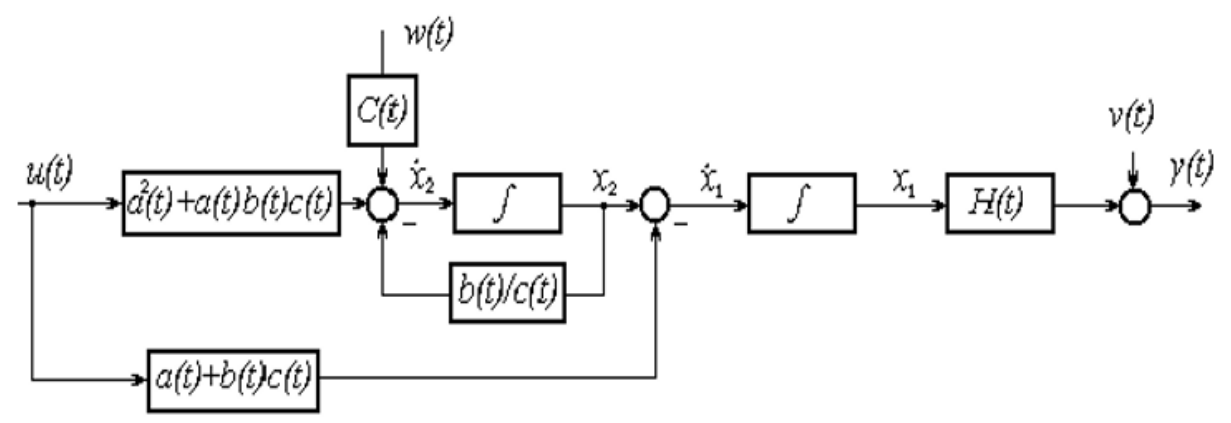

Fig. 1. The structural scheme of the system, defined in the state space.

Taking into account that the initial state of the system $x\left(t_{0}\right)$ is a random Gaussian vector with known mathematical expectation $M\left[x\left(t_{0}\right)\right]=\bar{x}_{0} \quad$ and the correlation matrix

$$
\operatorname{cov}\left[x\left(t_{0}\right), \bar{x}\left(t_{0}\right)\right]=M\left\{\left[x\left(t_{0}\right)-\bar{x}\left(t_{0}\right)\right]\left[x\left(t_{0}\right)-\bar{x}\left(t_{0}\right)\right]^{\mathrm{T}}\right\}=P\left(t_{0}, t\right)=P_{0},
$$

and also that the initial state of the system (2), random effects and measurement errors for all $t \geq t_{0}$ are mutually uncorrelated:

$$
\begin{gathered}
\operatorname{cov}\left[x\left(t_{0}\right), w(t)\right]=M\left\{\left[x\left(t_{0}\right)-\bar{x}\left(t_{0}\right)\right] w^{\mathrm{T}}(t)\right\}=0, \\
\operatorname{cov}\left[x\left(t_{0}\right), v(t)\right]=M\left\{\left[x\left(t_{0}\right)-\bar{x}\left(t_{0}\right)\right] v^{\mathrm{T}}(t)\right\}=0, \\
\operatorname{cov}[w(t), v(\tau)]=M\left[w(t) v^{\mathrm{T}}(\tau)\right]=0,
\end{gathered}
$$

we find a linear unbiased estimate of the vector $x(t)=\left[\begin{array}{l}x_{1} \\ x_{2}\end{array}\right]$, based on the results of observations $y(\tau),\left(t_{0} \leq \tau \leq t\right)$.

We denote this estimate by $z(t)=\hat{x}(t)$. Such an estimate can be obtained at the output of a filter described by a vector differential equation [1]:

$$
\dot{z}(t)=F(t)+G(t) u(t)+K(t) y(t)
$$

with estimation error

$$
e(t)=x(t)-z(t)
$$

In order for the process at the output of the filter to be unbiased, the equality should be

$$
M[\hat{x}(t)]=M[x(t)]=\bar{x}(t)
$$

Calculating the mathematical expectation of both sides of equation (4), we obtain:

$$
M[\dot{z}(t)]=F(t) M[z(t)]+G(t) u(t)+K(t) M[y(t)] .
$$


Then, taking into account that $M[y(t)]=H(t) M[x(t)]$, on the basis of (5), the differential equation for the average value of the state vector of the system will be written:

$$
\dot{\bar{x}}(t)=[F(t)+K(t) H(t)] \bar{x}(t)+G(t) u(t) .
$$

After calculating the mathematical expectation from both parts of equation (2), we obtain the equation for the average value of the state vector of the technological system $\dot{\bar{x}}=A(t) x(t)+B(t) u(t)$ or

$$
\left[\begin{array}{l}
\dot{x}_{1} \\
\dot{x}_{2}
\end{array}\right]=\left[\begin{array}{ll}
0 & 1 \\
0 & \frac{b(t)}{c(t)}
\end{array}\right]\left[\begin{array}{l}
\mathrm{x}_{1} \\
\mathrm{x}_{2}
\end{array}\right]+\left[\begin{array}{l}
-a(t)-b(t) c(t) \\
a^{2}(t)+a(t) b(t) c(t)
\end{array}\right] u(t) .
$$

Comparing (6) and (7), we write the first condition for unbiasedness of the state vector estimator with the help of the filter under consideration:

$$
F(t)=A(t)-K(t) H(t) ; \quad G(t)=B(t) .
$$

The second condition is that equations (5) and (6) are solved under the same initial condition:

$$
\bar{x}_{0}=M\left[\hat{x}\left(t_{0}\right)\right]=M\left[x\left(t_{0}\right)\right]=\bar{x}_{0} .
$$

If the unbiasedness conditions (8) and (9) are satisfied, then the filter equation (3) takes the form:

$$
\begin{aligned}
& {\left[\begin{array}{l}
\hat{\dot{x}}_{1} \\
\hat{\dot{x}}_{2}
\end{array}\right]=\left[\begin{array}{ll}
0 & 1 \\
0 & \frac{b(t)}{c(t)}
\end{array}\right]\left[\begin{array}{l}
\hat{\mathrm{x}}_{1} \\
\hat{\mathrm{x}}_{2}
\end{array}\right]+\left[\begin{array}{l}
-a(t)-b(t) c(t) \\
a^{2}(t)+a(t) b(t) c(t)
\end{array}\right] u(t)+} \\
& +\left[\begin{array}{l}
K_{1}(t) \\
K_{2}(t)
\end{array}\right]\left(y(t)-\left[\begin{array}{ll}
1 & 0
\end{array}\right]\left[\begin{array}{l}
\hat{\mathrm{x}}_{1} \\
\hat{\mathrm{x}}_{2}
\end{array}\right]\right) \\
& \mathrm{z}_{0}=\overline{\mathrm{x}}_{0}
\end{aligned}
$$

Graphical interpretation of the observed system and filter is shown in Fig. 2.

In expression (10), the matrix of filter intensity coefficients remains unknown $K(t)$, providing an optimal estimate, in which the components of the estimation error have a minimum variance.

The coefficient matrix of such a filter is given by [1]:

$$
K(t)=P(t) H^{T}(t) R^{-1}(t),
$$

$\begin{array}{rl}\text { where } & P(t) \quad-\quad \text { correlation matrix } \text { of estimation errors when } \\ M[e(t)]=0 & P(t)=M\left[e(t) e^{T}(t)\right] .\end{array}$

Since the initial value of the matrix $P(t)$ :

$$
P\left(t_{0}\right)=M\left\{\left[x\left(t_{0}\right)-\bar{x}_{0}\right]\left[x\left(t_{0}\right)-\bar{x}_{0}\right]^{T}\right\}
$$

and $z\left(t_{0}\right)=x_{0}$, then according to $(10) P\left(t_{0}\right)=P_{0}$. 


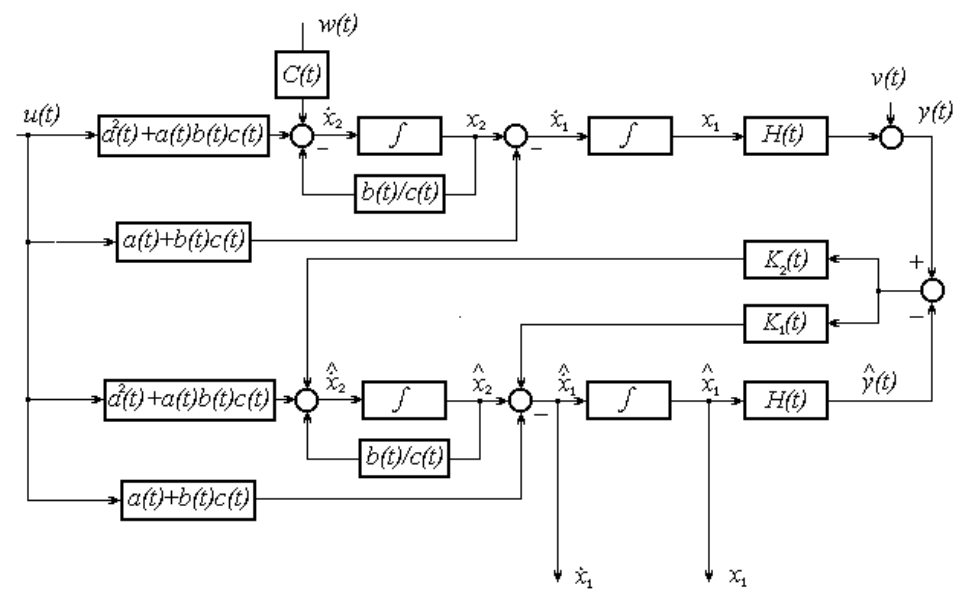

Fig. 2. Structural diagram of a stochastic Lunberger observer with a Kalman-Bussy filter

The correlation error matrix is a solution of the matrix differential Riccati equation [1]:

$$
\begin{aligned}
& \dot{P}(t)=A(t) P(t)+P(t) A^{T}(t)-P(t) H^{T}(t) R^{-1}(t) H(t) P(t)+ \\
& +C(t) Q(t) C^{T}(t)
\end{aligned}
$$

which should be solved under the initial conditions (11). When grinding products for each particular matrix mode $A, B, C$ and $H$ are constant, and matrixes $Q=M\left[W(t) W^{T}(\tau)\right]=D[W]$ and $R=M\left[V(t) V^{T}(\tau)\right]=D[V]$, describing the statistical characteristics of the change in temperature and surface roughness, are scalar quantities, therefore the technological system, determined by equations (2), is stationary (Fig. 3).

For such a system, the Kalman-Bussy filter equation takes the form:

$$
\begin{aligned}
& {\left[\begin{array}{l}
\hat{\dot{x}}_{1} \\
\hat{\dot{x}}_{2}
\end{array}\right]=\left[\begin{array}{ll}
0 & 1 \\
0 & \frac{b}{c}
\end{array}\right]\left[\begin{array}{l}
\hat{\mathrm{x}}_{1} \\
\hat{\mathrm{x}}_{2}
\end{array}\right]+\left[\begin{array}{c}
-a-b c \\
a^{2}+a b c
\end{array}\right] u(t)+\left[\begin{array}{l}
K_{1} \\
K_{2}
\end{array}\right]\left(y-\left[\begin{array}{ll}
1 & 0
\end{array}\right]\left[\begin{array}{l}
\hat{\mathrm{x}}_{1} \\
\hat{\mathrm{x}}_{2}
\end{array}\right]\right) .} \\
& \mathrm{z}_{0}=\overline{\mathrm{x}}_{0}
\end{aligned}
$$

Then the matrix of filter intensity coefficients is constant and is given by:

$$
\hat{K}=\hat{P} \hat{H} R^{-1},
$$

where $\hat{P}$ - a positive definite matrix that is a solution of the algebraic matrix Riccati equation:

$$
\left[\begin{array}{ll}
0 & 1 \\
0 & \frac{b}{c}
\end{array}\right] P+\hat{P}\left[\begin{array}{cc}
0 & 0 \\
1 & \frac{b}{c}
\end{array}\right]-\hat{P}\left[\begin{array}{l}
1 \\
0
\end{array}\right] D^{-1}(V)\left[\begin{array}{ll}
1 & 0
\end{array}\right] \hat{P}+\left[\begin{array}{l}
0 \\
\frac{1}{c}
\end{array}\right] D(W)\left[0 \frac{1}{c}\right]=0
$$




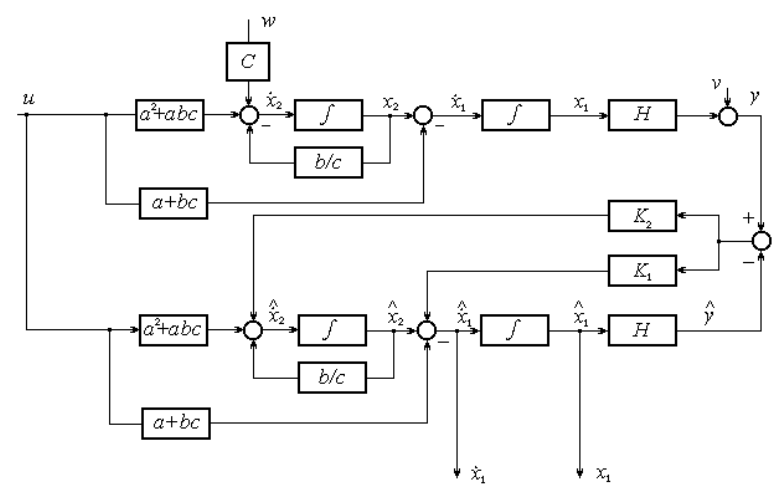

Fig. 3. Structural diagram of a stationary system with a Kalman-Bussy filter.

Hence the matrix $\hat{P}$ can be obtained as a steady-state solution of the differential equation:

$$
\dot{P}=\left[\begin{array}{ll}
0 & 1 \\
0 & \frac{b}{c}
\end{array}\right] P+P\left[\begin{array}{cc}
0 & 0 \\
1 & \frac{b}{c}
\end{array}\right]-P\left[\begin{array}{l}
1 \\
0
\end{array}\right] D^{-1}(V)[1 \quad 0] P+\left[\begin{array}{l}
0 \\
\frac{1}{c}
\end{array}\right] D(W)\left[\begin{array}{ll}
0 & \frac{1}{c}
\end{array}\right],
$$

T.e. $P=\lim _{t \rightarrow \infty} P(t)$.

The constancy of the matrix $\hat{K}$ makes it very simple to implement a family of linear program filters using a computer.

To build the Kalman-Bussy filter, a program has been developed that allows solving the Riccati equation of the object and the filter.

As an illustration of the program operation, the Kalman-Bussy filter coefficients were calculated for the stationary mode of the grinding process of the camshaft main journals with the following initial data: $G_{\kappa}=0,244 \cdot 10^{3} \mathrm{Ns} / \mathrm{m} ; C_{u \kappa}=35 \cdot 10^{6} \mathrm{~N} / \mathrm{m} ; C_{u}=18 \cdot 10^{6} \mathrm{~N} / \mathrm{m}$; $C_{\kappa}=11,89 \cdot 10^{6} \mathrm{~N} / \mathrm{m} ; \alpha=4 \cdot 10^{-6} ; \dot{S}_{y}=5 \cdot 10^{-6} \mathrm{~m} / \mathrm{s}, Q=D_{W}=4 \cdot 10^{-12} \mathrm{~m} / \mathrm{s}, R=D_{v}=0,36 \cdot 10^{-12}$, where the statistical data on the intensity of thermal deformations $Q$ and the value of the layer in which the roughness $R$ is distributed are taken from the works [5,12].

As a result of the program, numerical data characterizing the quality of the filter is shown on Fig. 4.

\section{Conclusion}

Analysis of the calculation results shows that the steady state of the filter comes in $0.4 \mathrm{~s}$ (corresponds to one revolution of the part) with readiness for practical use. This allows us to evaluate the state of the system in real time, and even in the case of measuring only one coordinate $Q_{\Sigma}$, the filter gives estimates of all coordinates, and the maximum estimation errors do not exceed $10 \%$. 


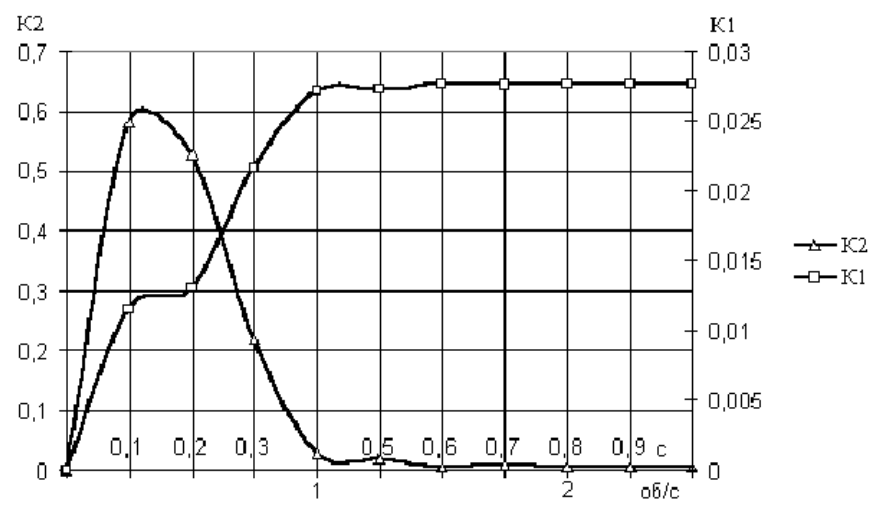

$a$

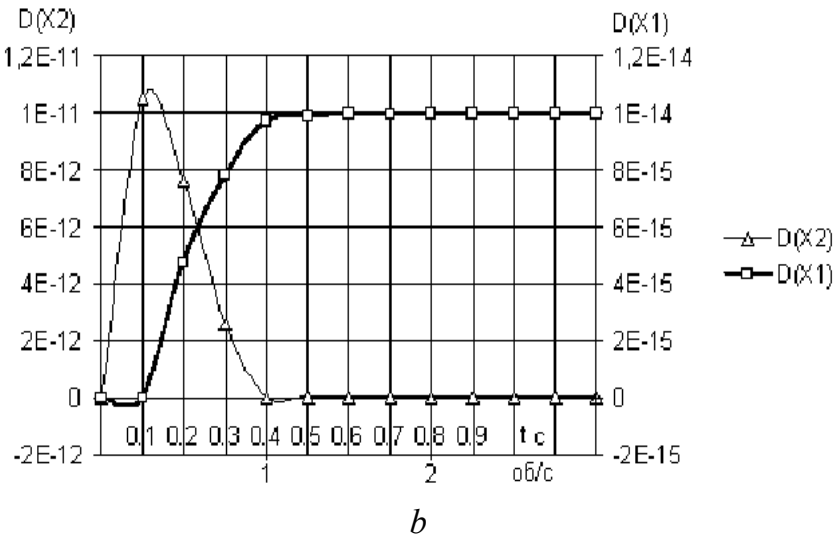

Fig. 4. Graphs of the dependences of the intensification filter on time (a) and the variance of the estimates on time (b)

\section{References}

1. Lobanov D.V., Yaniushkin A.S., Arkhipov P.V. Stress-strain state of carbide-tipped cutting elements with diamond sharpening, J. Sci. Tolyatti State University, v. 3-1 (331), pp. 85-91 (2015).

2. Lobanov D.V., Muliukhin N.V. A technique for predicting the damage of a hard alloy when sharpening a tool for processing nonmetallic composites, Actual problems in mechanical engineering, v. 5. №1-2, pp. 78-84 (2018).

3. Derusso P. The space of states in control theory (Nauka, Moscow, 1970).

4. Bratan S.M. Automatic control of machining processes (Vuzovskiy uchebnik: INFRAM, Moscow, 2017).

5. Bratan S.M. Improving the quality of parts when grinding in floating workshops (Vuzovskiy uchebnik: INFRA-M, Moscow, 2018).

6. Bratan S.M. Synthesis of the Kalman-Bussy filter for estimating the state of the grinding operation. J. Sci. Sevastopol State University, pp. 87-91 (2015).

7. Vladeckaya E.A. Ensuring the quality of grinding by reducing external disturbances in a floating workshop, J. Sci. FGBOU VO «PGU». v. 6 (314), pp.88-103 (2015). 
8. Vladeckaya E.A. Development of a shaping filter that simulates the dynamics of sea waves in a floating repair shop, J. Sci. Sevastopol State University. v. 150, pp. 36-40 (2014).

9. Harchenko A.O. Increase the accuracy of the round grinding process by efficient vibration isolation of the machine. International Scientific Conference on engineering design and research of automotive vehicles and machines «SAKON'08». - Rzeszow Przeclaw, Polska, pp. 47-58 (2008).

10. Harchenko A.O. The reaction of the dynamic system to an arbitrary signal, using the example of a machine in the conditions of a floating workshop. «Maszyny i procesy produkcyjne» (Mashiny i proizvodstvennye processy). Lublin, Polska: Politechnika Lubelska. pp. 86-98 (2015).

11. Bratan S.M. Investigation of the reliability of the vibration isolating device of the grinding machine. Naukoyemkiye tekhnologii $\mathrm{v}$ mashinostroyenii. J. Sci. Bryansk State University. v. №9 (63). pp. 10-15 (2016).

12. Vladetskaya E.A. Modeling of vibration effects from external sources when grinding parts in laboratory conditions. J. Sci. Sevastopol State University, v.4. pp. 25-34 (2016). 